\title{
THE FOUR-DIMENSIONAL PERFECT-MIRSKY CONJECTURE
}

\author{
JEREMY LEVICK, RAJESH PEREIRA, AND DAVID W. KRIBS
}

(Communicated by Pamela B. Gorkin)

\begin{abstract}
We verify the Perfect-Mirsky Conjecture on the structure of the set of eigenvalues for all $n \times n$ doubly stochastic matrices in the four-dimensional case. The $n=1,2,3$ cases have been established previously and the $n=5$ case has been shown to be false. Our proof is direct and uses basic tools from matrix theory and functional analysis. Based on this analysis we formulate new conjectures for the general case.
\end{abstract}

For over a century, stochastic and doubly stochastic matrices have been amongst the most well studied classes of matrices. Motivation has come from pure mathematics and from a wide variety of applications to fields such as economics, engineering, and quantum information.

In 1946, Dmitriev and Dynkin [3] considered the problem of characterizing the region, denoted by $\Theta_{n}$, given by the subset of the complex plane containing all eigenvalues of all $n \times n$ stochastic matrices. They reformulated the problem geometrically, noting its equivalence to finding $\lambda \in \mathbb{C}$ such that multiplication by $\lambda$ preserves some polygon in the complex plane. A few years later, Karpelevich [6] managed to completely solve the problem by expanding on Dmitriev and Dynkin's methods. He found implicit parametrizations of the boundary arcs for the regions $\Theta_{n}$. A detailed description of Karpelevich's result along with diagrams of the regions can be found in [8] or [1].

Sometime later, though almost fifty years ago still, Perfect and Mirsky [9] considered the analogous problem for doubly stochastic matrices; that is, they sought to characterize the region $\omega_{n} \subset \mathbb{C}$ containing all eigenvalues of all $n \times n$ doubly stochastic matrices. Let $\Pi_{k}$ denote the convex hull of the $k^{t h}$ roots of unity;

$$
\Pi_{k}=\left\{\sum_{j=1}^{k} t_{j} e^{\frac{2 \pi i j}{k}}: t_{j} \geq 0, \sum_{j} t_{j}=1\right\} .
$$

Perfect and Mirsky conjectured that $\omega_{n}=\bigcup_{k=1}^{n} \Pi_{k}$. They proved the conjecture for $n=1,2,3$, and left the cases $n \geq 4$ open.

Received by the editors May 29, 2013 and, in revised form, June 28, 2013 and November 11, 2013.

2010 Mathematics Subject Classification. Primary 15B51; Secondary 15A18, 46A55, 46H05.

Key words and phrases. Stochastic matrices, doubly stochastic matrices, eigenvalues, eigenvectors, linear operators. 
More recently, in 2007 Mashreghi and Rivard [7] exhibited a counterexample for $n=5$; in fact, they showed the matrix

$$
\left(\begin{array}{lllll}
0 & 0 & 0 & 1 & 0 \\
0 & 0 & \frac{1}{2} & 0 & \frac{1}{2} \\
0 & \frac{1}{2} & \frac{1}{2} & 0 & 0 \\
0 & \frac{1}{2} & 0 & 0 & \frac{1}{2} \\
1 & 0 & 0 & 0 & 0
\end{array}\right)
$$

has an eigenvalue that lies outside the $5 \times 5$ Perfect-Mirsky region. Thus, the Perfect-Mirsky conjecture is known to be true for $n=3$, and false for $n=5$. As the eigenvalues of the above matrix lie in $\omega_{6}$, the Perfect-Mirsky conjecture remains open for $n \geq 6$.

We note that the Karpelevich and Perfect-Mirsky regions coincide for $n=3$ but differ for all higher $n$. In particular, $\Theta_{3}=\bigcup_{k=1}^{3} \Pi_{k}$ but $\Theta_{n} \supsetneq \bigcup_{k=1}^{n} \Pi_{k}$ for $n \geq 4$, and hence the Karpelevich approach cannot be readily extended to the setting of doubly stochastic matrices. It should be noted that Perfect and Mirsky's proof that $\omega_{3}=\bigcup_{k=1}^{3} \Pi_{k}$ in [9, Theorem 12] works for stochastic matrices as well as doubly stochastic matrices and hence provides another proof for the $n=3$ Karpelevich result that $\Theta_{3}=\bigcup_{k=1}^{3} \Pi_{k}$.

We also note that while the Karpelevich and Perfect-Mirsky regions differ when $n \geq 4$, Karpelevich's result shows us that the two line segments with one endpoint at 1 and the other at $\pm e^{\frac{2 \pi i}{n}}$ form part of the boundary of $\Theta_{n}$ and hence are also part of the boundary of $\omega_{n}$. Hence the Perfect-Mirsky conjecture always holds locally near one. In [4, Johnson gives an improvement of this local result for doubly stochastic matrices having certain zero patterns. The same author has also studied some conditions under which certain stochastic matrices are similar to doubly stochastic matrices [5], which gives further connections between the stochastic and doubly stochastic eigenvalue problems. We will not use these results here, and so we refer the interested reader to the original papers for details.

In this paper we verify the Perfect-Mirsky conjecture for $n=4$. Our proof uses basic tools from matrix theory and functional analysis. We have covered the relevant background material above. The main result and preliminary results are contained in the following section, as are new conjectures on the structure of these fundamental eigenvalue sets.

We can identify $\mathbb{C}$ as a real vector space isomorphic to $\mathbb{R}^{2}$. We note that $\Pi_{4}$ is the unit ball of the $l^{1}$ vector norm on $\mathbb{R}^{2}$. We remind the reader of the wellknown formula for the $l^{1}$ operator norm on the space of $n \times n$ matrices $\|A\|_{1}=$ $\max _{1 \leq j \leq n} \sum_{i=1}^{n}\left|a_{i j}\right|$.

We begin by representing vectors in $\mathbb{C}^{n}$ as convex polygons in the complex plane.

Definition 1. Let $v=\left(v_{1}, \ldots, v_{n}\right) \in \mathbb{C}^{n}$. Let $K_{i}(v) \subseteq \mathbb{C}, i=1,2$, be the convex hulls:

$$
K_{1}(v)=\operatorname{conv}\left\{v_{i}\right\}_{i=1}^{n}, \quad K_{2}(v)=\operatorname{conv}\left\{v_{j}+v_{k}\right\}_{1 \leq j<k \leq n}
$$

The set of all eigenvalues of $n \times n$ row stochastic matrices corresponding to the eigenvector $v$ can be described in terms of $K_{1}(v)$ in a natural way.

Proposition 1. Let $v \in \mathbb{C}^{n}$ and let $\lambda \in \mathbb{C}$. Then there exists an $n \times n$ row stochastic matrix $A$ such that $A v=\lambda v$ if and only if $\lambda K_{1}(v) \subseteq K_{1}(v)$.

Proof. We note that if $A v=\lambda v$, then for all $j, \lambda v_{j}=\sum_{k=1}^{n} a_{j k} v_{k}$ and hence $\lambda v_{j} \in$ $K_{1}(v)$. Since all extreme points of $\lambda K_{1}(v)$ lie in $K_{1}(v)$, we have $\lambda K_{1}(v) \subseteq K_{1}(v)$. The converse direction follows from reversing these steps. 
Corollary 1. Let $K \subseteq \mathbb{C}$ be a convex m-gon. Let $\lambda \in \mathbb{C}$ with $\lambda K \subseteq K$. Then $\lambda$ is the eigenvalue of an $m \times m$ stochastic matrix.

We require Birkhoff's theorem [2], which we now state for completeness.

Theorem 1 (Birkhoff). The set of $n \times n$ doubly stochastic matrices is the convex hull of the $n \times n$ permutation matrices.

Birkhoff's theorem can be used to show the relationship between doubly stochastic matrices and $K_{2}$.

Corollary 2. Let $v \in \mathbb{C}^{n}$ and let $A$ be an $n \times n$ doubly stochastic matrix. Then $K_{2}(A v) \subseteq K_{2}(v)$.

Proof. Let $w=A v$. The extreme points of $K_{2}(w)$ are of the form $w_{j}+w_{k}$, where $j, k$ are integers with $1 \leq j<k \leq n$. Hence it is enough to show that $w_{j}+w_{k} \in K_{2}(v)$. Since $A$ is in the convex hull of the permutation matrices, $w_{j}+w_{k}$ is in the convex hull of the set $\left\{[P v]_{j}+[P v]_{k}: P\right.$ is a permutation matrix $\}$, which is $K_{2}(v)$.

Before proving the next result, we need a definition and a preliminary geometric lemma.

Definition 2. Let $v=\left\{v_{1}, v_{2}, \ldots, v_{n}\right\} \in \mathbb{C}^{n}$. If no $v_{i}$ is in the convex hull of the other $\left\{v_{j}\right\}_{j \neq i}$, we say that the entries of $v$ are convexly independent.

Lemma 1. Let $C$ be a convex polygon, let $v$ be one of its vertices and let $l$ be a line that runs though $v$. Let $H$ be one of the open half-planes whose boundary is $l$. If $H$ contains any vertices of $C$, it must contain at least one vertex of $C$ adjacent to $v$.

We note that if $v$ is an eigenvector of a doubly stochastic matrix corresponding to an eigenvalue other than one, then the sum of the entries of the eigenvector is zero. We now show that $K_{2}(v)$ is a convex $n$-gon when $v \in \mathbb{C}^{n}$ and the entries of $v$ are convexly independent and $\sum_{k=1}^{n} v_{k}=0$.

Proposition 2. Let $v \in \mathbb{C}^{n}$ and suppose the entries of $v$ are convexly independent and $\sum_{k=1}^{n} v_{k}=0$. Without loss of generality, we list them in clockwise order: $v_{1}, v_{2}, \ldots, v_{n}$. Then we have

$$
K_{2}(v)=\operatorname{conv}\left\{v_{j}+v_{j+1}\right\}_{j=1}^{n},
$$

where we have set $v_{n+1}=v_{1}$.

Proof. We will show that $z$ is an extreme point of $K_{2}(v)$ if and only if $z=v_{j}+v_{j+1}$.

Let $v_{j}$ and $v_{j+1}$ be two adjacent vertices of $K_{1}(v)$. Then $\left[v_{j}, v_{j+1}\right]$ is a face of $K_{1}(v)$ and lies on a supporting hyperplane $L$. Let $L$ be defined by the equation $f(x)=b$, where $b$ is a positive real number and $f(z)$ is a real linear functional $f(z)=\operatorname{Re}(a z)$. Then $f\left(v_{j}\right)=f\left(v_{j+1}\right)=b>0$. All other vertices of the polygon lie on the same side of $L$ as 0 , and so $f\left(v_{k}\right)<b$ for $k \neq j, j+1$. Then $f\left(v_{j}+v_{j+1}\right)=$ $2 b>f\left(v_{k}+v_{m}\right)$ for any $(k, m) \neq(j, j+1)$, and so $v_{j}+v_{j+1}$ is an extreme point of $K_{2}(v)$.

Next we show the converse. Suppose $v_{j}+v_{k}$ is an extreme point of $K_{2}(v)$; then let $f$ be a real linear functional on $\mathbb{C}$ which achieves its maximum on $K_{2}(v)$ at $v_{j}+v_{k}$. If $f\left(v_{j}\right)=f\left(v_{k}\right)$, then the two vertices lie on the same proper face of $K_{1}(v)$ and hence must be adjacent. So suppose $f\left(v_{j}\right) \neq f\left(v_{k}\right)$, and without loss of generality let $f\left(v_{j}\right)>f\left(v_{k}\right)$. Since $v_{j}$ is the only vertex of $K$ contained in the 
open half-plane $\left\{z: f(z)>f\left(v_{k}\right)\right\}, v_{j}$ must be adjacent to $v_{k}$ by Lemma 1 and the result follows.

Let $e=(1,1,1,1)^{T}$ and $A$ be a four-by-four doubly stochastic matrix. If $v$ is an eigenvector of $A$ corresponding to an eigenvalue other than one, then $e^{T} v=$ $\left(e^{T} A\right) v=e^{T}(A v)=\lambda e^{T} v$. Therefore $e^{T} v$, which is also the sum of the entries of the eigenvector $v$, is zero. In this case

$$
K_{2}(v)=\operatorname{conv}\left\{z_{1}, z_{2},-z_{1},-z_{2}\right\}
$$

where $z_{1}=v_{1}+v_{2}=-v_{3}-v_{4}$ and $z_{2}=v_{2}+v_{3}=-v_{4}-v_{1}$.

Proposition 3. Let $z_{1}$ and $z_{2}$ be complex numbers with $z_{1} \neq 0$ and let $K=$ conv $\left\{z_{1}, z_{2},-z_{1},-z_{2}\right\}$. If $\lambda$ is a complex number such that $\lambda K \subset K$, then $\lambda \in \Pi_{4}$.

Proof. Let $K$ be as above and let $\lambda$ be a complex number such that $\lambda K \subset K$. If $K$ is a line segment, then $\lambda$ must be a real number in $[-1,1]$. So suppose $K$ is a quadrilateral. Similarly if $\lambda$ is a real number, $\lambda \in[-1,1]$. So suppose $\lambda=a+b i$ with $b \neq 0$. Let $M=\left(\begin{array}{cc}a & -b \\ b & a\end{array}\right)$ be the multiplication operator by $\lambda$. We note that $\lambda \in \Pi_{4}$ if and only if $\|M\|_{1} \leq 1$. Let $S$ be the invertible real linear transformation which maps $\Pi_{4}$ to $K$ (by mapping $e_{1}$ to $z_{1}$ and $e_{2}$ to $z_{2}$ ). Then $\left\|S^{-1} M S\right\|_{1} \leq 1$ since $M$ maps $K$ to $K$. We now complete the proof by showing that $\|M\|_{1} \leq\left\|S^{-1} M S\right\|_{1}$ for all invertible two-by-two real matrices $S$. Since $S^{-1} M S$ is a real matrix with the same trace as $M$, there exists $x, y, x \in \mathbb{R}$ such that

$$
M=\left(\begin{array}{cc}
a+x & y \\
z & a-x
\end{array}\right) .
$$

Since $a^{2}-x^{2}-y z=\operatorname{det}\left(S^{-1} M S\right)=\operatorname{det}(M)=a^{2}+b^{2}$ it follows that $-y z=x^{2}+b^{2}$. Therefore we get

$$
\begin{aligned}
\left\|S^{-1} M S\right\|_{1} & =\max \{|a+x|+|z|,|y|+|a-x|\} \\
& \geq \frac{1}{2}(|a+x|+|a-x|+|y|+|z|) \\
& \geq|a|+\sqrt{|y z|}=|a|+\sqrt{x^{2}+b^{2}} \\
& \geq|a|+|b|=\|M\|_{1} .
\end{aligned}
$$

We now verify the Perfect-Mirsky conjecture for $n=4$.

Theorem 2. The set of all eigenvalues of $4 \times 4$ doubly stochastic matrices is $\bigcup_{k=1}^{4} \Pi_{k}$.

Proof. Let $\lambda$ be the eigenvalue of a $4 \times 4$ doubly stochastic matrix and let $v$ be the corresponding eigenvector. If the entries of $v$ are convexly dependent, then $v$ is the eigenvalue of a $3 \times 3$ stochastic matrix by Corollary 1 and we have $\lambda \in \bigcup_{k=1}^{3} \Pi_{k}$. If the entries of $v$ are convexly independent, then $\lambda K_{2}(v) \subseteq K_{2}(v)$ by Corollary 2, In this case, $K_{2}(v)$ is of the form $\operatorname{conv}\left\{z_{1}, z_{2},-z_{1},-z_{2}\right\}$ by Proposition 2 and hence $\lambda \in \Pi_{4}$, and this completes the proof.

The considerations above lead us to formulate the following conjecture on the regions $\omega_{n}$.

Conjecture 1. The region $\omega_{n}=\Theta_{n-1} \cup \Pi_{n}$. 
This conjecture is identical to the Perfect-Mirsky conjecture when $n \leq 4$; exactly the cases for which the Perfect-Mirsky conjecture is known to be true. This conjecture is also compatible with Mashreghi and Rivard's counterexample; the eigenvalue they present is well within the relevant region of the $4 \times 4$ Karpelevich region, as our conjecture would suggest.

We note that if the entries of the eigenvector are not convexly independent, then Corollary 1 implies that the corresponding eigenvector lies in $\Theta_{n-1}$. This is the case with the Mashreghi-Rivard counterexample, where the eigenvector associated with the eigenvalue outside $\Pi_{5}$ is not convexly independent. We suspect that all counterexamples to the Perfect-Mirsky Conjecture are eigenvalues which correspond to eigenvectors having convexly dependent entries; we also state this as a conjecture in its own right.

Conjecture 2. Let $A$ be an $n \times n$ doubly stochastic matrix. Let $\lambda$ be an eigenvalue of $A$. If the entries of an eigenvector corresponding to $\lambda$ are convexly independent, then $\lambda \in \bigcup_{k=1}^{n} \Pi_{k}$.

Finally, we give a conjecture relating the eigenvalue regions of the stochastic and doubly stochastic matrices.

Conjecture 3. Any eigenvalue of an $n-1 \times n-1$ stochastic matrix is also the eigenvalue of some $n \times n$ doubly stochastic matrix.

We note that one can prove Conjecture 1 by proving both Conjecture 2 and Conjecture 3 .

\section{ACKNOWLEDGEMENTS}

The contents of this paper form part of the first author's doctoral thesis. The authors would like to thank Pal Fischer for several useful and interesting discussions. The authors would like to thank the referees for many helpful suggestions. The first author was partly supported by a University of Guelph Graduate Scholarship. The second author was partly supported by NSERC Discovery Grant 400550. The third author was partly supported by NSERC Discovery Grant 400160.

\section{REFERENCES}

[1] Luca Benvenuti and Lorenzo Farina, Eigenvalue regions for positive systems, Systems Control Lett. 51 (2004), no. 3-4, 325-330, DOI 10.1016/j.sysconle.2003.09.009. MR2037263 (2004k:93055)

[2] Garrett Birkhoff, Three observations on linear algebra (Spanish), Univ. Nac. Tucumán. Revista A. 5 (1946), 147-151. MR0020547(8,561a)

[3] N. Dmitriev and E. Dynkin, On characteristic roots of stochastic matrices (Russian, with English summary), Bull. Acad. Sci. URSS. Sér. Math. [Izvestia Akad. Nauk SSSR] 10 (1946), 167-184. MR0017269 (8,129f)

[4] Charles R. Johnson, An inclusion region for the field of values of a doubly stochastic matrix based on its graph, Aequationes Math. 17 (1978), no. 2-3, 305-310. MR0491769 (58 \#10968)

[5] Charles R. Johnson, Row stochastic matrices similar to doubly stochastic matrices, Linear and Multilinear Algebra 10 (1981), no. 2, 113-130, DOI 10.1080/03081088108817402. MR618581 (82g:15016)

[6] F. I. Karpelevič, On characteristic roots of matrices with nonnegative coefficients, Uspehi Matem. Nauk (N.S.) 4 (1949), no. 5(33), 177-178. MR0031462 (11,154b)

[7] Javad Mashreghi and Roland Rivard, On a conjecture about the eigenvalues of doubly stochastic matrices, Linear Multilinear Algebra 55 (2007), no. 5, 491-498, DOI 10.1080/03081080600899296. MR 2363549(2008m:15058) 
[8] Henryk Minc, Nonnegative matrices, Wiley-Interscience Series in Discrete Mathematics and Optimization, John Wiley \& Sons Inc., New York, 1988. A Wiley-Interscience Publication. MR 932967 (89i:15001)

[9] Hazel Perfect and L. Mirsky, Spectral properties of doubly-stochastic matrices, Monatsh. Math. 69 (1965), 35-57. MR.0175917 (31 \#193)

Department of Mathematics and Statistics, University of Guelph, Guelph, Ontario, CANADA N1G 2W1

Department of Mathematics and Statistics, University of Guelph, Guelph, Ontario, CANADA N1G 2W1

Department of Mathematics and Statistics, University of Guelph, Guelph, Ontario, CANAdA N1G 2W1 